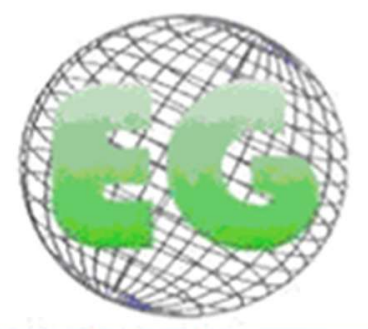

ISSN 1695-6141 $\mathbf{N}^{\circ} 63$

\title{
ORIGINALES
}

\section{Incidencia, características y medidas aplicadas en pacientes con gripe A (H1N1) en el contexto hospitalario durante el periodo 2016- 2018}

Incidence, characteristics and measures applied in patients with influenza A virus (H1N1) in the hospital context during the 2016-2018 period

\author{
Marina Ignacio-Torres ${ }^{1}$ \\ Ana Hornero-López ${ }^{2}$ \\ Emilio Jiménez-Martínez ${ }^{2}$ \\ Jordi Adamuz ${ }^{3}$
}

\begin{abstract}
${ }^{1}$ Servicio de Urgencias del Hospital Universitario de Bellvitge, Instituto de Investigación Biomédica de Bellvitge (IDIBELL). Barcelona. España. marina.ignaciotorres@gmail.com

2 Departamento de Control de Infección. Hospital Universitario de Bellvitge, Instituto de Investigación Biomédica de Bellvitge (IDIBELL). Barcelona. España.

3 Departamento de Gestión del Conocimiento y Sistemas de Información en Enfermería. Hospital Universitario de Bellvitge, Instituto de Investigación Biomédica de Bellvitge (IDIBELL). Barcelona. España.
\end{abstract}

\section{https://doi.org/10.6018/eglobal.446241}

Recibido: 24/09/2020

Aceptado: $13 / 01 / 2021$

\section{RESUMEN:}

Introducción: El virus de la gripe ocasiona anualmente epidemias estacionales con amplia extensión mundial y se estima que entre el 5 y el $20 \%$ de la población padece gripe cada año. En abril de 2009 se confirmaron los primeros casos de infección humana causados por un nuevo virus de la gripe $A$ (H1N1).

Objetivo y Método: En el período de epidemia (semana 40-20) del 2016-2018 se realizó un estudio observacional descriptivo prospectivo multicéntrico en dos hospitales públicos del área metropolitana sur de Barcelona con el objetivo de determinar la incidencia de pacientes positivos en gripe $A(H 1 N 1)$, así como las características demográficas y clínico-evolutivas de estos pacientes, la temporalidad en la detección del virus y medidas aplicadas por control de la infección.

Resultados: Los resultados del presente trabajo indican que la incidencia acumulada de Gripe A durante el periodo de estudio fue de 233,68 casos por cada 100.000 habitantes. Casi el $90 \%$ de los pacientes padecía antecedentes patológicos siendo los más prevalentes la patología cardíaca y respiratoria. Además, el $40 \%$ de los pacientes hospitalizados presentó complicaciones, principalmente la neumonía. El tratamiento y días de aislamiento fueron según los estándares recomendados.

Conclusión: Estos hallazgos muestran la elevada incidencia de gripe $\mathrm{A}$, así como los beneficios de que los equipos de control de la infección realicen el seguimiento del cumplimiento del tratamiento y medidas para evitar la transmisión.

Palabras clave: Virus de la Influenza A, Infección, virología, prevalencia, N1H1. 


\begin{abstract}
:
Introduction:The influenza virus causes seasonal epidemics worldwide and it is estimated that between 5 and $20 \%$ of the population suffers from influenza each year. The first cases of human infection caused by a new influenza A virus (H1N1) were confirmed in April 2009.

Objective and Method: In the epidemic period (week 40-20) of 2016-2018, a multicenter prospective descriptive observational study was carried out in two public hospitals in the southern metropolitan area of Barcelona in order to determine the incidence of positive patients in influenza $A(H 1 N 1)$, as well as the demographic and clinical-evolutionary characteristics of these patients, the timing of virus detection and measures applied for infection control.

Results: The results of the present work indicate that the cumulative incidence of Influenza A during the study period was 233,68 per 100,000 inhabitants. Almost $90 \%$ of the patients had an underlying chronic condition, mostly heart disease and respiratory pathology. Furthermore, $40 \%$ of the patients presented complications, mainly pneumonia. Treatment and days of isolation were according to standard recommendations.

Conclusion: These findings show the high incidence of influenza A virus $(\mathrm{N} 1 \mathrm{H} 1)$ as well as the benefits that apports the infection control teams surveillance, monitoring the compliance with treatment and days of measures to avoid the transmission.
\end{abstract}

Key words: Influenza Virus A, Infection, Virology, prevalence, N1H1

\title{
INTRODUCCIÓN
}

La gripe es un importante problema de salud tanto por la mortalidad que puede provocar como por las complicaciones que puede ocasionar y los costes económicos y sociales que origina ${ }^{(1)}$.

En abril de 2009 se confirmaron los primeros casos de infección humana causados por un nuevo virus de la gripe $A(\mathrm{H} 1 \mathrm{~N} 1)$. El virus causante de la nueva pandemia era el resultado de una recombinación entre un virus porcino americano reordenado de 1997-1998, que a su vez contenía segmentos de virus porcino, aviar y humano, y un virus porcino de Euroasia que contenía segmentos de virus aviar ${ }^{(2)}$.

El 25 de abril de 2009 la Organización Mundial de la Salud (OMS) emite su primer comunicado donde advierte la presencia de un brote epidémico por un nuevo virus de gripe $\mathrm{A}(\mathrm{N} 1 \mathrm{H} 1)$ en EEUU y México, declarando una emergencia de salud pública de importancia internacional. El 27 de abril, la OMS elevó el nivel de alerta pandémica a la fase 4, tras verificar una transmisión interhumana capaz de causar brotes a nivel comunitario. El 29 de abril, la OMS cambia la fase de 4 a 5, considerando que hay casos con transmisión entre personas en dos países de una misma región sanitaria: EEUU y México. El 11 de junio de 2009 la OMS elevó la alerta por la gripe A, a nivel 6 , el máximo de la escala que configura una pandemia, tras comprobar la transmisión elevada y sostenida del virus en el mundo. Este hecho no ocurría desde 1968 con el Influenzavirus A subtipo H3N2, conocida como gripe de Hong Kong ${ }^{(3)}$.

La gripe A (H1N1), a finales de septiembre de 2009, afectó a nivel mundial a más de 343.298 personas produciendo 4.108 muertes (3). La incidencia de casos hospitalizados a nivel nacional con confirmación de gripe fue de 36 por 100.000 habitantes; 9 ingresos por cada mil casos de síndrome gripal diagnosticados, un $8 \%$ de los cuales causaron estancia en unidades de cuidados intensivos. La mortalidad fue de 17 casos por cada 100.000 habitantes, pero entre los casos de pacientes ingresados en las unidades de cuidados intensivos se elevó a más del $20 \%(1,4)$. En uno de los hospitales públicos catalanes durante el período pandémico se atendió en 
el servicio de urgencias a 1.144 pacientes con sospecha clínica de gripe $A(H 1 N 1)$ de los cuales, un 44,8\% (513 pacientes) requirieron ingreso ${ }^{(2)}$.

Según estudios previos, alrededor el $26 \%$ de los casos confirmados fueron hospitalizados. Como factores de riesgo de complicaciones, además de la edad avanzada, aparece el embarazo, asma, patología pulmonar, patología cardíaca, inmunodeficiencia y obesidad, como factores con mayor impacto ${ }^{(5)}$.

Diversos estudios muestran la necesidad de unidades de control de la infección en los hospitales para prevenir y detectar precozmente posibles brotes de gripe $A$ entre pacientes y profesionales en períodos pandémicos ${ }^{(5,6)}$.

Por otro lado, la evidencia muestra que los sistemas de vigilancia realizados por los servicios de control de la infección y las campañas de vacunación deben continuar después del período pandémico ${ }^{(7)}$, además de la necesidad de continuar investigando sobre la incidencia y las características de los pacientes afectados por este virus.

\section{OBJETIVOS}

\section{Objetivo principal}

Determinar la incidencia de pacientes positivos en Gripe A (H1N1) en dos hospitales de tercer nivel en el período de epidemia 2016-2018.

\section{Objetivos específicos}

Determinar las características demográficas y clínico-evolutivas de los pacientes afectados por el virus de la Gripe A (H1N1).

Conocer la temporalidad en la detección de la Gripe $\mathrm{A}(\mathrm{H} 1 \mathrm{~N} 1)$ en los pacientes de los dos hospitales incluidos en el estudio.

Describir las medidas aplicadas por la unidad de control de la infección del Hospital Universitario de Bellvitge a los pacientes positivos en Gripe $\mathrm{A}(\mathrm{H} 1 \mathrm{~N} 1)$.

\section{METODOLOGÍA}

El estudio se llevó a cabo en dos hospitales de tercer nivel situados en el área metropolitana sud de Barcelona. Ambos centros son referencia para los municipios de Hospitalet y el Prat de Llobregat y centro de referencia terciaria territorial para todo el eje Sud de Cataluña. Disponen de todas las especialidades, incluida la oncología, excepto la atención materno-infantil.

Se realizó un estudio cuantitativo observacional descriptivo prospectivo des de la semana 40 del año 2016 hasta la semana 20 del 2018. Durante el período pandémico, el equipo de control de la infección del centro realiza un seguimiento exhaustivo de los nuevos casos de gripe A, estableciendo medidas de tratamiento, seguimiento y recomendación de cuidados respecto aislamiento mientras el paciente se encuentra hospitalizado y elabora anualmente un informe con estos datos. 
Los criterios de inclusión fueron: pacientes diagnosticados del virus de la gripe $A$ (H1N1) con PCR positiva en el período de estudio y que el diagnóstico de la gripe se realizara a través de PCR-RT por frotis faríngeo, aspirado traqueal o broncoaspirado de secreciones bronquiales (BAS). Para la selección de la muestra se utilizó muestreo de tipo no probabilístico consecutivo durante el periodo de estudio de todos los pacientes que cumplieran criterios de inclusión.

La obtención de los datos se realizó de forma prospectiva mediante un seguimiento diario realizado por el servicio del control de la infección. Las variables se recogieron a partir de la historia clínica electrónica mediante la plataforma SAP ${ }^{\circ}$ (Systems, Applications \& Products, Waldorff,Germany). Los principales datos recogidos fueron: datos sociodemográficos (género, edad) y variables clínico-evolutivas (antecedentes patológicos, índice de Charlson, días de ingreso, días en la detección de gripe, método de obtención del frotis, diagnóstico de alta, fecha PCR, tratamiento, días de tratamiento, unidad de hospitalización, días de aislamiento y complicaciones durante el ingreso). Se consideró que el paciente era portador del virus de la gripe A (H1N1) si durante el período de estudio presentó un PCR positiva analizada en los laboratorios de nuestros centros hospitalarios participantes.

Este estudio no precisó de consentimiento informado ya que el seguimiento de pacientes con gripe forma parte de la práctica habitual del centro. Se cumplieron los principios bioéticos y de las normativas reguladoras actuales en materia de investigación clínica y los principios establecidos en la Declaración de Helsinki y sus enmiendas posteriores. El tratamiento, la comunicación y la cesión de los datos de carácter personal de todos los sujetos participantes se ajustaron a la normativa legal vigente. Los datos se anonimizaron mediante un código numérico consecutivo. Este estudio fue aprobado por el Comité Ético de Investigación Clínica de los centros participantes con código PR091/17.

El análisis de los datos se ha efectuado con el programa IBM SPSS versión 25.0. Se ha realizado un análisis descriptivo de todas las variables. Las variables de ajuste fueron analizadas en función del tipo de variable y presentadas en tablas. Las variables categóricas nominales fueron descritas por medio del número de casos, el porcentaje respecto al total por categoría y el número de datos ausentes. Las variables continuas que seguían una distribución normal se describieron por medio del número de casos, la media y la desviación estándar. Las variables continuas que no seguían una distribución normal son descritas por medio del número de casos, la mediana, el primer y el tercer cuartil.

\section{RESULTADOS}

Durante el periodo de estudio, se admitieron 146.353 pacientes de los cuales 341 fueron positivos para Gripe A, siendo la incidencia acumulada durante los dos períodos de estudio de 233,68 casos por cada 100.000 habitantes. En el primer período de estudio entre las semanas 40 y 20 de los años 2016-2017 la incidencia fue de 124,35 casos por cada 100.000 habitantes. En el segundo periodo de 2017-2018 la incidencia fue de 109,33 casos por cada 100.000 habitantes. 
Se observa que los meses donde se detectaron mayor número de casos fue entre diciembre-febrero, tal y como muestran estudios previos $^{(8)}$. (ver Gráfico 1)

Gráfico 1. Gráfico de tendencias de la Gripe A (N1H1) periodo 2016-2018.

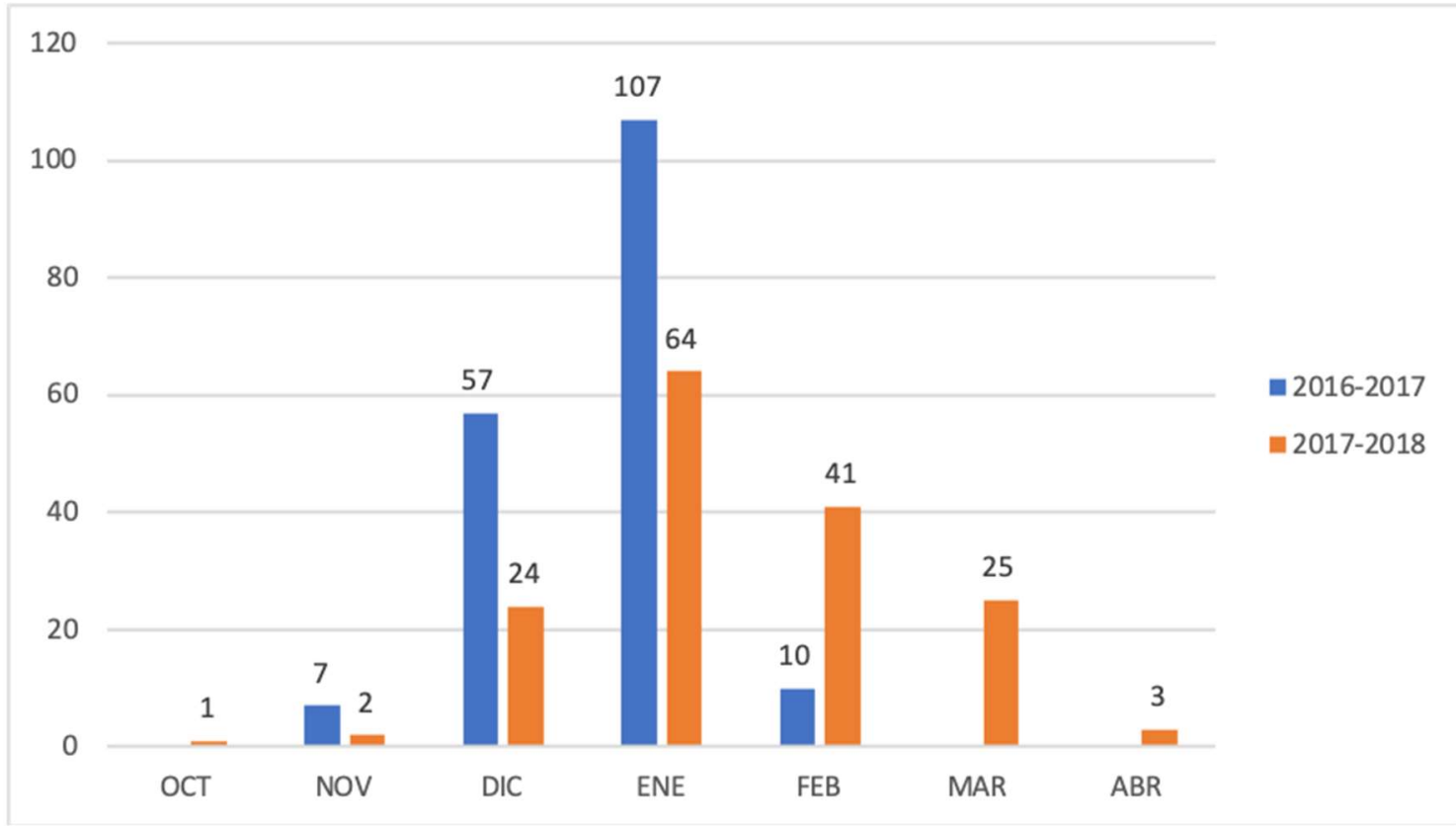

Entre las 341 determinaciones de PCR positivas, el método mayoritario de detección fue el frotis faríngeo en un $95 \%$ de los casos, seguido del exhudado/pus nasal en un $4,4 \%$ de los casos y finalizando con el método BAS en un $0,6 \%$.

Del total de la muestra, un $55,7 \%(n=190)$ de los pacientes fueron hombres y la media de edad fue de 67 años (Desviación Estándar [DE]=16.8). La mediana de días de hospitalización fue de 5 días (IQR: 3-10). En cuanto a los antecedentes patológicos, los resultados de este estudio determinan que un $89,7 \%$ de ellos tenían alguna comorbilidad asociada. La patología cardíaca ha sido el antecedente más prevalente en un $42,2 \%$ de los pacientes, la insuficiencia renal crónica en un $26,4 \%$, la diabetes en un $24,1 \%$. La obesidad $(19,1 \%)$, la patología pulmonar $(17,3 \%)$ y el asma $(8,2 \%)$ fueron comorbilidades menos prevalentes (tabla 1). En base a los antecedentes que padecían los pacientes estudiados, se ha determinado que la mediana del índice de Charlson en los pacientes con Gripe A N1H1 positiva es de 2 puntos (Rango interquartil $[I Q R]=1.00 ; 3.00$ ). 
Tabla 1. Datos socio-clínicos de los pacientes afectos por el virus N1H1: Datos según la unidad de obtención de la muestra

\begin{tabular}{|c|c|c|c|c|}
\hline & $\begin{array}{l}\text { TOTAL } \\
\mathrm{N}=\mathbf{3 4 1}\end{array}$ & $\begin{array}{l}\text { Urgencias } \\
\mathrm{N}=293\end{array}$ & $\begin{array}{l}\text { Otras } \\
\text { unidades } \\
\mathrm{N}=\mathbf{3 8}\end{array}$ & $\begin{array}{l}\text { Hospitalización } \\
\mathrm{N}=10\end{array}$ \\
\hline $\begin{array}{l}\text { Edad, Media (desviación } \\
\text { estándar) }\end{array}$ & $67.0(16.8)$ & $69.0(16.2)$ & $51.1(14.0)$ & $69.5(9.6)$ \\
\hline \multicolumn{5}{|l|}{ Género, $\mathbf{N}(\%)$ : } \\
\hline Hombre & $190(55.7 \%)$ & $164(56.0 \%)$ & $19(50.0 \%)$ & $7(70.0 \%)$ \\
\hline Mujer & $151(44.3 \%)$ & $129(44.0 \%)$ & $19(50.0 \%)$ & $3(30.0 \%)$ \\
\hline AP, $\mathbf{N}(\%)$ & $306(89.7 \%)$ & 274 (93.5\%) & $22(57.9 \%)$ & $10(100 \%)$ \\
\hline Cardiopatía, N (\%) & $144(42.4 \%)$ & $136(46.6 \%)$ & $4(10.5 \%)$ & $4(40.0 \%)$ \\
\hline Diabetes Mellitus, N (\%) & $82(24.1 \%)$ & $78(26.7 \%)$ & $2(5.3 \%)$ & $2(20.0 \%)$ \\
\hline Inmunodeficiencia, N (\%) & $20(5.9 \%)$ & $18(6.2 \%)$ & $2(5.3 \%)$ & $0(0.0 \%)$ \\
\hline Insuficiencia renal, $\mathbf{N}(\%)$ & $90(26.4 \%)$ & $82(28.0 \%)$ & $5(13.2 \%)$ & $3(30.0 \%)$ \\
\hline Insuficiencia Hepática, N (\%) & $17(5.0 \%)$ & $16(5.5 \%)$ & $0(0.0 \%)$ & $1(10.0 \%)$ \\
\hline Obesidad, $\mathbf{N}(\%)$ & 65 (19.1\%) & $56(19.1 \%)$ & $6(15.8 \%)$ & $3(30.0 \%)$ \\
\hline $\begin{array}{l}\text { Patología neuromuscular, } \mathrm{N} \\
\text { (\%) }\end{array}$ & $11(3.2 \%)$ & $10(3.4 \%)$ & $1(2.6 \%)$ & $0(0.0 \%)$ \\
\hline $\begin{array}{l}\text { Patología pulmonar crónica, N } \\
\text { (\%) }\end{array}$ & 59 (17.3\%) & $58(19.8 \%)$ & $0(0.0 \%)$ & $1(10.0 \%)$ \\
\hline Asma, $\mathbf{N}(\%)$ & $28(8.2 \%)$ & $25(8.5 \%)$ & $2(5.3 \%)$ & 1 (10.0\%) \\
\hline
\end{tabular}

En cuanto a las complicaciones que han padecido los pacientes afectos por Gripe A $\mathrm{N} 1 \mathrm{H} 1$ en el período del estudio, destacar que un $41,1 \%$ de los pacientes han presentado algún tipo de complicación. De entre las complicaciones más prevalentes destaca la neumonía en un $13,2 \%$ de los casos y la insuficiencia respiratoria en un $12,9 \%$, precisando ingreso en $\mathrm{UCl}$ en un $5,9 \%$. (tabla 2 )

Tabla 2. Principales complicaciones de la Gripe A (N1H1):

\begin{tabular}{|c|c|}
\hline & Todos $\mathrm{N}=341$ \\
\hline Complicaciones, $\mathbf{N}(\%)$ & $140(41.1 \%)$ \\
\hline UCl, N (\%) & $20(5.9 \%)$ \\
\hline $\begin{array}{l}\text { Insuficiencia Respiratoria } \\
\text { N (\%) }\end{array}$ & $44(12.9 \%)$ \\
\hline Neumonía, N (\%) & 45 (13.2\%) \\
\hline VMI, N (\%) & $11(3.2 \%)$ \\
\hline Exitus, $\mathbf{N}(\%)$ & $20(5.9 \%)$ \\
\hline $\begin{array}{l}\text { Sobreinfección } \\
\text { respiratoria, } \mathbf{N}(\%)\end{array}$ & $33(9.7 \%)$ \\
\hline
\end{tabular}

En relación con la temporalidad en la detección del Virus $\mathrm{N} 1 \mathrm{H} 1$, un $86,5 \%$ de los casos fueron confirmados a través del frotis con una PCR positiva en las primeras $48 \mathrm{~h}$ des que el paciente consultó en el hospital. El restante 13,5\% se consideraron casos nosocomiales. Cabe destacar que, durante el período de estudio, se administró el tratamiento específico a un $76,9 \%$ de los pacientes confirmados de Gripe A en las 
primeras $48 \mathrm{~h}$, con una mediana de 4 días con medidas de precaución de transmisión por gotas $(I Q R=3-6)$.

\section{DISCUSIÓN}

Según el plan de información de las infecciones respiratorias agudas en Cataluña (PIDIRAC), en el periodo 2016-2017 la tasa de incidencia fue de 64,79 casos por cada 100.000 habitantes ${ }^{(8)}$. En nuestro estudio, la incidencia entre los pacientes que consultaron a urgencias es mayor, obteniendo unos resultados de 124,35 casos por cada 100.000 habitantes. En el periodo 2017-2018, la incidencia fue de 95,62 casos por cada 100.000 h habitantes ${ }^{(8)}$, en la misma línea, en este estudio se obtuvo una tasa de incidencia de 109,33 casos por cada 100.000 habitantes.

Los resultados del presente trabajo indican que la incidencia acumulada de Gripe A durante el período de estudio de 233,68 casos por cada 100.000 habitantes, resultados por encima de la incidencia global de Cataluña publicados en el informe PIDIRAC, que publican como incidencia global de ambos periodos 160,41 casos por cada 100.00 habitantes.

Cabe destacar que, tal y como refieren otros estudios no existen prácticamente diferencias en la afectación por géneros (9). En relación con los antecedentes patológicos de interés, nuestro estudio muestra que aproximadamente un $90 \%$ de los pacientes padecía algún antecedente, dato por encima de los estudios previos, que muestran un porcentaje alrededor del $70 \%{ }^{(10)}$. Los antecedentes de etiología cardíaca resultan mayores en incidencia en nuestro estudio, seguido de la patología respiratoria y renal. Este dato coincide con los resultados de antecedentes de origen pulmonar publicados por Álvarez Lerma F, et al., con un porcentaje de afectados del $17,9 \%{ }^{(11)}$. Aun así, este dato es controvertido ya que no coincide con otros estudios publicados anteriormente donde la cifra aproximada de pacientes con factores de riesgo rodeaba el $65 \%{ }^{(5)}$ y el antecedente más prevalente era la obesidad con una incidencia del $34.8 \%{ }^{(12)}$.

En cuanto a las complicaciones las respiratorias, la neumonía y la insuficiencia respiratoria son las más prevalentes. Estos resultados van en línea con la publicación de González. R et al. donde se muestra que las complicaciones respiratorias son las que presentan con mayor incidencia, presentándose en un $64,3 \%$ de los casos ${ }^{(4)}$. Este dato confirma los resultados de Álvarez Lerma $\mathrm{F}$ et al donde más del $34 \%$ de los pacientes precisaron de soporte ventilatorio de algún tipo ${ }^{(11)}$.

La detección de los pacientes afectos de gripe A se realizó en un $86,5 \%$ de los casos en las primeras $48 \mathrm{~h}$ des de la consulta del paciente al hospital por sintomatología gripal, siendo este un diagnostico inmediato y, se administró el tratamiento específico con antivirales en un $76,9 \%$ de los pacientes. Estos números concuerdan con la literatura ya publicada por otros autores de una confirmación diagnóstica con una mediana de dos días des del inicio de los síntomas y una administración ligeramente mayor de antivirales correspondiente al $87 \%$ de los pacientes ${ }^{(4)}$. Tal como consta en la bibliografía consultada, el tratamiento debería iniciarse en el momento de tener una muestra positiva y se aconseja una duración de 5 días ${ }^{(3)}$. 
Los pacientes que precisaron medidas de precaución de transmisión por gotas estuvieron aislados una mediana de 4 días. Este hecho es debido a que gran parte de los pacientes fueron dados de alta por estabilidad clínica antes de cumplir los 5 días establecidos. Puesto que el periodo de pandemia de gripe estacional se concentra en unas semanas concretas (8) es clave para la optimización de los recursos hospitalarios, para evitar el colapso de los servicios asistenciales y contener la transmisión hospitalaria del virus; un equipo formado en control de infección que gestione y coordine de forma eficiente las medidas de precaución y los días necesarios, el cohorting de pacientes y pueda detectar si hay algún brote de gripe nosocomial. Los centros deben apostar por esta filosofía de trabajo e invertir recursos, tanto materiales como humanos potenciando la figura de la enfermera de control de la infección, especializada tanto en control como en la prevención de las infecciones relacionadas con la asistencia sanitaria.

\section{CONCLUSIONES}

Los hallazgos de este estudio confirman que el hecho de padecer antecedentes patológicos predispone en cierta medida a la infección por el virus influenza A H1N1. Por otro lado, la incidencia de Gripe A en el periodo de estudio es de 233,68 casos por cada 100.000 habitantes y las complicaciones aparecen en un $41,1 \%$ de los afectados. Estos datos muestran la importancia de disponer de un equipo de control de infección formado por enfermeras que sean capaces de hacer un manejo eficiente del paciente con síntomas gripales durante el periodo pandémico, para evitar de esta forma el contagio en el medio hospitalario y optimizar los recursos.

\section{REFERENCIAS}

1. Marin J. Et al. Pacientes con gripe por el virus influenza A (H1N1) pdm09 ingresados en la UCI. Impacto de las recomendaciones de la SEMICYUC. Med Intensiva. 2018;42(8):473-481.

2. Lera E, Wörner NT, Sancosmed M,et al. Impacto del virus de la gripe A (H1N1) 2009 en un servicio de urgencias de un hospital de tercer nivel. An Pediatr.2011; 75(1):13-20.

3. Moreno MC, Estrada JM, Fernández D, López A. Actualización sobre el virus de la gripe $A$ : experiencia de una pandemia des de la perspectiva enfermera. Metas de Enferm.2010;13(5):8-16.

4. González R, Balasso V, Uriona S. Et al. Características de los pacientes y carga assistencial durante la pandèmia de gripe A (H1N1) 2009 en el Hospital Universitario Vall d'Hebron de Barcelona. Enferm Infecc Microbiol Clin. 2011;29 (8): 593-600.

5. Trilla A. Transmisión de la gripe en los servicios de urgencias. Emergencias. 2018;30:5-6.

6. Mendoza JL, Quirós V, Jiménez M. Et al. Impacto de la transmisión nosocomial del virus de la gripe en un hospital de agudos. Rev Esp Salud Pública. 2018; 92:1-7.

7. Viasus D, Antón A, Pumarola T and Carratalà J, Influenza A(H1N1)pdm09: beyond the pandemic. Enferm Infecc Microbiol Clin. 2012;30(Supl 4):54-57.

8. Generalitat de Catalunya. Pla d'informació de les infeccions respiratòries agudes a Catalunya (PIDIRAC) [Internet]. 12.08.2019 [revisado; consultado]. Disponible en: 
http://canalsalut.gencat.cat/ca/professionals/vigilancia-epidemiologica/pladinformacio-de-les-infeccions-respiratories-agudes-a-catalunya-pidiracl

9. Zhoua,F. Et al. Risk factors for nosocomial infection among hospitalised severe influenza $\mathrm{A}(\mathrm{H} 1 \mathrm{~N} 1)$ pdm09 patients. Respiratory Medicine.2018;134 (1): 86-91.

10. Bouneb, R. Et al. Characteristics and outcome of ill critical patients with influenza A infection. The Pan African Medical Journal. 2018; 29:174-182.

11. Alvarez Lerma F. Et al. Delay in Diagnosis of Influenza A (N1H1) pdm09 virus infection in critically ill patients and impact on clinical outcome. 2016; 20(1):337-348.

12. Alvarez Lerma $F$, et al. Characteristics of patients with hospital-acquired influenza A (H1N1) pdm09 virus admitted to the intensive care unit. Journal of Hospital Infection.2017; 95 (1):200-206. 